\title{
SOX9 Expression Predicts Relapse of Stage II Colon Cancer Patients
}

Espersen, Maiken Lise Marcker; Linnemann, Dorte; Christensen, lb Jarle; Alamili, Mahdi; T. Troelsen, Jesper; Høgdall, Estrid

Published in:

Human Pathology

DOI:

10.1016/j.humpath.2015.12.026

Publication date:

2016

Document Version

Peer reviewed version

Citation for published version (APA):

Espersen, M. L. M., Linnemann, D., Christensen, I. J., Alamili, M., T. Troelsen, J., \& Høgdall, E. (2016). SOX9 Expression Predicts Relapse of Stage II Colon Cancer Patients. Human Pathology, 52, 38-46.

https://doi.org/10.1016/j.humpath.2015.12.026

\section{General rights}

Copyright and moral rights for the publications made accessible in the public portal are retained by the authors and/or other copyright owners and it is a condition of accessing publications that users recognise and abide by the legal requirements associated with these rights.

- Users may download and print one copy of any publication from the public portal for the purpose of private study or research.

- You may not further distribute the material or use it for any profit-making activity or commercial gain.

- You may freely distribute the URL identifying the publication in the public portal.

Take down policy

If you believe that this document breaches copyright please contact rucforsk@kb.dk providing details, and we will remove access to the work immediately and investigate your claim. 


\section{Accepted Manuscript}

SOX9 Expression Predicts Relapse of Stage II Colon Cancer Patients

Maiken Lise Marcker Espersen MSc, Dorte Linnemann MD, DMSc, Ib Jarle Christensen MSc, Mahdi Alamili MD, PhD, Jesper T. Troelsen PhD, DMSc, Estrid Høgdall PhD, DMSc

PII:

S0046-8177(16)00028-9

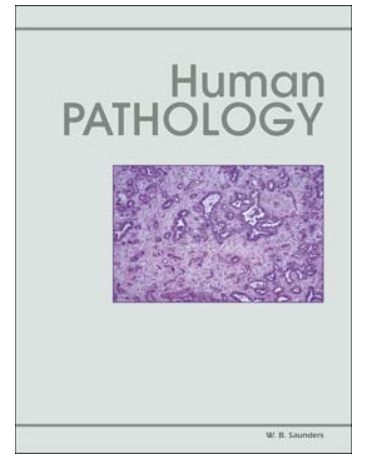

DOI: doi: 10.1016/j.humpath.2015.12.026

Reference: YHUPA 3790

To appear in: $\quad$ Human Pathology

Received date: 19 October 2015

Revised date: 13 December 2015

Accepted date: 19 December 2015

Please cite this article as: Espersen Maiken Lise Marcker, Linnemann Dorte, Christensen Ib Jarle, Alamili Mahdi, Troelsen Jesper T., Høgdall Estrid, SOX9 Expression Predicts Relapse of Stage II Colon Cancer Patients, Human Pathology (2016), doi: 10.1016/j.humpath.2015.12.026

This is a PDF file of an unedited manuscript that has been accepted for publication. As a service to our customers we are providing this early version of the manuscript. The manuscript will undergo copyediting, typesetting, and review of the resulting proof before it is published in its final form. Please note that during the production process errors may be discovered which could affect the content, and all legal disclaimers that apply to the journal pertain. 
Title: SOX9 Expression Predicts Relapse of Stage II Colon Cancer Patients

Running Head: SOX9 predicts relapse of stage II colon cancer patients

Authors: Maiken Lise Marcker Espersen MSc ${ }^{\mathrm{a}, \mathrm{b}}$, Dorte Linnemann MD, DMSc ${ }^{\mathrm{a}}$, Ib Jarle

Christensen $\mathrm{MSc}^{\mathrm{a}}$, Mahdi Alamili MD, $\mathrm{PhD}^{\mathrm{c}}$, Jesper T. Troelsen PhD, DMSc ${ }^{\mathrm{b}}$, Estrid Høgdall PhD,

$\mathrm{DMSc}^{\mathrm{a}}$

\section{Affiliations}

${ }^{\mathrm{a}}$ Department of Pathology, Herlev University Hospital, Herlev Ringvej 75, DK-2730 Herlev,

Denmark.

${ }^{\mathrm{b}}$ Department of Science, Systems and Models, Roskilde University, Universitetsvej 1, DK-4000

Roskilde, Denmark.

${ }^{\mathrm{c}}$ Department of Surgery, Køge University Hospital, Lykkebækvej 1, DK-4600 Køge, Denmark.

\section{Email addresses}

Maiken Lise Marcker Espersen (maiken.lise.marcker.espersen@regionh.dk)

Dorte Linnemann (dorte.linnemann@regionh.dk)

Ib Jarle Christensen (ib.jarle.christensen@regionh.dk)

Mahdi Alamili(mahdi_alamili@hotmail.com)

Jesper T. Troelsen (troelsen@ruc.dk)

\section{Corresponding Author}

Estrid Høgdall

Email: estrid.hoegdall@regionh.dk

Molecular Unit, Department of Pathology, Herlev University Hospital, Herlev Ringvej 75, DK-

2730 Herlev, Denmark

Telephone: +45 38689132, Fax: +4544883711

\section{Conflicts of interests}

The authors declare no conflicts of interest. 


\section{Abstract}

The aim of this study was to investigate if the protein expression of Sex-determining region y-box 9 (SOX9) in primary tumors could predict relapse of stage II colon cancer patients.144 patients with stage II primary colon cancer were retrospectively enrolled in the study. SOX9 expression was evaluated by immunohistochemistry and mismatch repair status was assessed by both immunohistochemistry and promoter hyper-methylation assay. High SOX9 expression at the invasive front was significantly associated with lower risk of relapse when including the SOX9 expression as a continuous variable (from low to high expression) in univariate (HR: 0.73 ; 95\% CI: $0.56-0.94 ; p=0.01)$ and multivariate cox proportional hazards analysis (HR: $0.75 ; 95 \% \mathrm{CI}: 0.58$ $0.96 ; p=0.02$ ) adjusting for mismatch repair deficiency and histopathological risk factors. Conversely, low SOX9 expression at the invasive front was significantly associated with high risk of relapse, when including SOX9 expression dichotomous variable, in univariate (HR: 2.32; 95\% CI: $1.14-4.69 ; p=0.02$ ) and multivariate analysis (HR: $2.32 ; 95 \%$ CI: $1.14-4.69 ; p=0.02$ ) adjusting for histopathological risk factors and mismatch repair deficiency. In conclusion high levels of SOX9 of primary stage II colon tumors predict low risk of relapse whereas low levels of SOX9 predict high risk of relapse. SOX9 may have an important value as a biomarker when evaluating risk of relapse for personalized treatment.

\section{Keywords}

SOX9 Transcription Factor, Biomarkers, Colon Cancer, Recurrence, Mismatch Repair Deficiency 


\section{Introduction}

Colorectal cancer is one of the most frequent malignancies of the western world[1]. Approximately one fourth of patients with colon cancer are diagnosed with stage II colon cancer[2]. Today, a minor group of patients with stage II colon cancer is offered adjuvant therapy based on high-risk histopathological features (T4 stage, vein invasion, perineural invasion, margin involvement, number of sampled lymph nodes, perforation, and low differentiated histology)[2,3].

Despite proper surgical intervention approximately $20 \%$ of all patients with stage II colon cancer have relapse of their cancer. Yet still no optimal biomarker has been established in the clinic to identify high risk patients and predict relapse of stage II colon cancer. Thus, the incentive for novel prognostic and predictive markers to identify the patients, who most likely will benefit from additional treatment, is extensive.

One of the hallmarks of cancer is genomic instability[4]. Microsatellite instability (MSI) can occur as a consequence of mismatch repair deficiency (dMMR)[5]. Germline mutations in mismatch repair (MMR) genes are associated with the Lynch Syndrome whereas MutL homolog 1 (MLH1) promoter hyper-methylations are primarily found in sporadic colon cancer cases[6].

The cancer stem cell theory proposes that cancer stem cells are involved in initiation, progression, reoccurrence of cancer, and treatment response. Sex-determining region y-box 9 (SOX9) is a transcription factor involved in several developmental processes and is important for cell proliferation, senescence, and lineage commitment[7-10]. We hypothesized that there may be an association between the SOX9 expression in primary tumors of patients with stage II colon cancer and their risk of relapse. To test our hypothesis we investigated the SOX9 expression by immunohistochemistry (IHC) in primary tumors of patients with stage II colon cancer. 


\section{Methods and materials}

\subsection{Patient cohort}

The study was performed as a retrospective cohort study. Formalin-fixed paraffin-embedded (FFPE) primary tumors from 144 patients diagnosed and treated for stage II colon cancer at Glostrup University Hospital, Gentofte University Hospital, and Herlev University Hospital in Denmark, were included consecutively from January 2005 to August 2008 using the national pathology registry of Denmark and patient medical records. The patients had undergone complete surgical resection as primary treatment. The inclusion period was based on the desire of a follow-up period of at least 5 years. Registered data, inclusion and exclusion criteria can be found in Appendix A. Enrollment and exclusion of patients is shown in Figure 1. The study was approved by the Scientific Ethics Committee of the Capital Region of Denmark (H-1-2013-028) and by the Data Protection Agency of the Capital Region of Denmark (2007-58-0015).

\subsection{Tumor Tissue}

The tumor tissue had been processed as part of the diagnostic routine following curative surgery. The tumor tissue was fixated in $10 \%$ neutral buffered formalin for $\geq 48$ hours prior to paraffin embedding. From each patient two tissue blocks were obtained of the primary tumor. Full slides of the tumor were used for IHC against SOX9. Tissue microarrays (TMA's) were constructed with four $1 \mathrm{~mm}$ cores from each secondary tissue block as previously described[11]. A fifth $1 \mathrm{~mm}$ core in a tumor cell enriched area ( $\geq 50 \%$ tumor cells) was punched for DNA extraction.

\subsection{IHC analysis}

All analysis and assays where conducted blinded to patient outcome.

\subsubsection{SOX9 IHC and evaluation}

$3 \mu \mathrm{m}$ slides were cut and incubated for $45 \mathrm{~min}$. at $60^{\circ} \mathrm{C}$. IHC was performed using the EnVision ${ }^{\mathrm{TM}}$ FLEX, High pH detection system (Dako, Denmark) and the automated Autostainer Link 48 (Dako, 
Denmark) according to manufacturer's instructions. Anti-SOX9 antibody $(1+10,000$, Merck Millipore) was used for SOX9 detection. Specification of the antibody can be found in Supplementary Table 1. The tissue slides were counterstained with Mayers hematoxylin using the automated slide stainer Tissue-Tek ${ }^{\circledR}$ Prisma ${ }^{\circledR} /$ Film $^{\circledR}$ (Sakura, Denmark). Finally, the slides were scanned using the Nanozoomer 2.0-HT (Hamamatzu, Germany).

The stability of the SOX9 antigen was evaluated by staining normal colon tissue that had been subjected to formalin-fixation for 3, 27, 51, and 123 hours. A control slide was included for each run and consisted of normal tissue from the colon, small intestine, testis, ventricle, and breast. The selection of the control tissues were based on previous reports of SOX9 staining[12-17].

SOX9 expression was evaluated at the invasive front of the tumor and at the luminal surface independently by a specialized pathologist and a trained molecular biologist supervised by a specialized pathologist. We defined the invasive front as the area where the tumor periphery invades deepest into the colonic tissue. The luminal surface refers to the luminal surface of the neoplastic glands.

Five random areas were selected for each region, using the image analysis software Visiopharm Integrator System (version 4.5.6.516, Visiopharm, Denmark). Percent positive and negative tumor nuclei were counted and given a score: score 0 ( $0-5 \%$ positive nuclei), score $1(>5-25 \%$ positive nuclei), score 2 ( $>25-50 \%$ positive nuclei), score 3 ( $>50-75 \%$ positive nuclei), and score 4 ( $>75 \%$ positive nuclei). An intensity score was given corresponding to score 0 (absent staining), score 1 (low), score 2 (moderate), and score 3 (high). The final score was generated by multiplying percent positive tumor nuclei with the intensity score. Tumors with overall score 0 was rerun to confirm the score and the few positive stromal cells was used as an internal control of the staining. Since SOX9 was present in almost all tumor nuclei when the staining was positive, the overall SOX9 expression was considered low when the overall score was $0-4$. An overall score ranging from $>4-12$ was 
considered as high SOX9 expression. A consensus score was generated in cases of inter-observer disagreement by evaluating the slides once more.

All histological features were evaluated by a specialized pathologist as part of the diagnostic routine. The criteria for vein infiltration were based on remnants of smooth muscle of the vein in the tumor tissue. Differentiation of the tumors was defined according to WHO classification of tumors, defining low differentiated tumors as tumors with $0-49 \%$ of glandular structures and high differentiated tumors by $>49 \%$ of glandular structures[18].

\subsubsection{MMR IHC status}

$3 \mu \mathrm{m}$ TMA sections was stained with monoclonal antibodies against MLH1 (Ready-To-Use, Dako, Denmark), MutS protein homolog 2 (MSH2) $(1+400$, CellMarque, CA, USA), MutS homolog 6 (MSH6) (Ready-To-Use, Dako, Denmark), and Postmeiotic Segregation Increased 2 (PMS2) (Ready-To-Use, Dako, Denmark) using the EnVision detection system described above. Specifications of the antibodies can be found in Supplementary Table 1. The tumor was considered dMMR if one or more of the four MMR proteins were absent in the tumor nuclei. If the tumor nuclei stained positive for one or more of the four MMR proteins, the tumor was MMR proficient (pMMR). Nuclear staining of stromal cells was used as an internal control. A positive control slide containing normal tonsil, appendix, pancreas, and liver was included in each run.

\subsection{Promoter hyper-methylation assay}

DNA was extracted from the $1 \mathrm{~mm}$ core as previously described[19]. Promoter hyper-methylation of $M L H 1, M S H 2, M S H 6$, and PMS2 was assessed by the SALSA MS-MLPA ME011 Mismatch Repair genes kit (cat. no. EK1-FAM, MRC-Holland, The Netherlands) and performed according to manufacturer's instructions. Using the enclosed software, the methylation ratio was analyzed as "hyper-methylated" or "unmethylated". All unmethylated cases were analyzed twice to confirm status of case. 


\subsection{Statistics}

The primary end point of the study was whether the protein expression of SOX9 in primary tumors from stage II colon cancer patients could predict relapse. A secondary endpoint was to investigate the prognostic value of dMMR in stage II colon cancer patients with relapse.

If the tumor was positive for either of the risk factors: T4 stage, nerve infiltration, vein infiltration, low differentiated histological appearance (unless the tumor was dMMR), tumor perforation, or less than 12 lymph nodes sampled at primary resection, the patient was grouped as having a histopathological risk factor. This was done due to the relative small numbers of some of the histopathological risk factors.

Association between SOX9 expression levels and age were explored by the Spearman rank correlation. Rank test for location (Mann-Whitney U and Kruskal-Wallis) was applied to investigate associations between SOX9 expression levels and categorical variables.

Survival probabilities were estimated by Kaplan-Meier plots and compared by the log-rank test. Univariate and multivariate analysis adjusting for the histopathological risk factor variable and dMMR was done using the Cox proportional hazards model containing the SOX9 expression as a dichotomous variable (high/low) or as a continuous variable with a hazard ratio of three differences in scores and with relapse as outcome. The model assumptions were validated by sensitivity testing and martingale residuals.

A 5-year overall survival univariate model with SOX9 expression at the invasive front and the luminal surface as a dichotomous variable was conducted. Weighted Cohen's Kappa statistics was applied for investigating inter-observer agreement. All statistical analysis was conducted in IBM SPSS Statistics 22 (IBM, Armonk, N.Y., USA) and SAS (version 9.3, SAS Institute, Cary, N.C., USA). $p$-values of $\leq 0.05$ were considered significant. 


\section{Results}

\subsection{Patients and clinical characteristics}

The median follow-up period was 92 months (range: 69-111 months). Among the 144 patients included in the study 37 patients (25.7\%) relapsed during follow-up and 58 patients (40.3\%) had died (Figure 1). The median time to relapse was 21 months (range: 4-84 months). Of the patients that relapsed $31(83.8 \%)$ had died. The majority of patients with relapse (24 patients, $64.9 \%$ ) had recurrence in two or more sites.

Overall $70(48.6 \%)$ of the patients had one or more histopathological risk factors associated with their primary tumor. All of the patients had a tumor cell free resection margin. The luminal surface of the tumor was not available from two patients. Thus, the luminal surface was only analyzed on tumor samples from 142 patients. Overall patient characteristics are presented in Table 1.

A total of 33 (22.9\%) patients had dMMR tumors, whereof 30 (90.9\%) were MLH1 and PMS2 deficient. MSH2 and MSH6 deficiency was seen in 1 (3.0\%) tumor and $2(6.1 \%)$ tumors demonstrated solely MSH6 deficiency. Promoter hyper-methylation assay was conducted to further characterize the patient cohort as most sporadic colon cancer cases are caused by $M L H 1$ promoter hyper-methylations[6]. Of the dMMR tumors 28 (84.8\%) were $M L H 1$ promoter hyper-methylated. No promoter hyper-methylation was observed in tumors with MSH2 and/or MSH6 deficiency. Overall 5 out of 144 (3.5\%) patients had dMMR tumors without promoter hyper-methylations.

\subsection{SOX9 expression}

The SOX9 staining was not was not affected by the fixation or the storage time of the tissue. The SOX9 expression in the normal tissue can be found in Supplementary Figure 1. Both intratumoral and intertumoral heterogeneous SOX9 expression was observed in the colon cancer tissue (Figure 2). In most cases SOX9 stained positive in $>75 \%$ (score 4 ) of the tumor nuclei, however, the staining intensity ranged from highly intense to very low. Interestingly, SOX9 protein expression 
was not detected in $11(7.6 \%)$ and $12(8.3 \%)$ of the tumors at the invasive front and luminal surface, respectively (Figure 2).

\subsection{SOX9 and risk of relapse}

There was no significant correlation between SOX9 expression at either the invasive front or at the luminal surface of the tumors and age, gender, tumor side, or histopathological risk factors (Table 1). Kaplain-Meier survival plots showed a significant difference in relapse free survival of patients with low SOX9 expression at the invasive front of their primary tumor compared to patients with high SOX9 expression (Figure 3). SOX9 expression levels at the luminal surface showed no significant difference in relapse free survival time (Figure 3).

Univariate cox proportional hazards analysis showed that low SOX9 expression at the invasive front in the tumor was statistically associated with higher risk of relapse, see Table 2. Similarly in multivariate analysis, low SOX9 expression at the invasive front was significantly associated with 2.3 higher risk relapse (Table 2). For univariate and multivariate survival analyses, including SOX9 as a continuous variable, the risk of relapse decreased with $25 \%$ when there was a difference of 3 in the SOX9 score from low SOX9 expression to high expression at the invasive front (Table 2).

For univariate analysis tumor perforation was not included due to the low number of events in the patient cohort. Of the histopathological risk factors T-stage, nerve infiltration, or less than 12 sampled lymph nodes increased the risk of relapse significantly with 2 to 4 -fold (Table 2). In multivariate analysis the occurrence of one or more histopathological risk factors was associated with an approximate 3-fold risk of relapse (Table 2). Furthermore, dMMR was significantly associated with an approximately $80 \%$ reduced risk of relapse both in univariate and multivariate analysis (Table 2). There was no association between low SOX9 expression and 5-year overall survival by univariate cox proportional hazards analysis (HR: $1.56 ; 95 \% \mathrm{CI}: 0.79-3.09 ; p=0.2$ ). The 
inter-observer agreement of the intensity score of SOX9 at the invasive front showed a high concordance with a Cohen's weighted k-coefficient of 0.84 (95\% CI: 0.77-0.91).

\section{Discussion}

To our knowledge this is the first study investigating the protein expression of SOX9 as a potential biomarker of relapse in patients diagnosed with a stage II colon cancer. By investigating a comprehensive characterized patient cohort with stage II colon cancer and following the REMARK guidelines[20], we found that low SOX9 expression at the invasive front of their primary tumors was significantly associated with relapse.

The SOX9 expression pattern was very heterogeneous in the tumors, especially in the central tumor region. In effort to adjust for the heterogeneity, the luminal surface and the invasive front within each tumor were scored separately. Our results emphasize the importance of histopathology in diagnostics and validation of the biomarker expression patterns. The inter-observer concordance was high even with two evaluators with different professions, indicating that this analysis is robust, reproducible, and could be implemented as part of the clinical routine.

The underlying molecular mechanisms for the association of decreased SOX9 expression with risk of relapse are not fully solved. Together with SOX8 and SOX10, SOX9 is part of the SOX family group called SOXE[21]. The function of the SOXE transcription factors are highly context dependent and they bind and regulate differently depending on the environment they are subjected to. Therefore, the regulation of SOX9 and the exact function of SOX9 is complex. The transcription factor has been reported as having both oncogenic and tumor-suppressive effects in colorectal cancer[7,9,10,22].

A recent paper by The Cancer Genome Atlas provided a comprehensive molecular analysis of colorectal carcinomas and reported that SOX9 was frequently mutated[23]. The mutations consisted of frameshift and nonsense mutations, suggesting that SOX9 may play a tumor-suppressive 
role[23]. This has further been supported by studies reporting widespread hyperplasia and dysplasia in the intestinal epithelium of Sox 9 deficient mice[10,24]. Additionally, SOX9 has been reported to be a canonical Wnt/ $\beta$-catenin target gene in human colon cancer cells [9], exerting a negative feedback loop by inhibiting the signaling pathway as well[10]. Abdel-Samad and colleagues reported a truncated variant of SOX9 lacking its transactivation domain. The variant stimulated the canonical Wnt pathway but also inhibited wildtype SOX9[25]. This could be a plausible explanation for the conflicting roles of SOX9 reported in the literature.

A low or non-detectable expression of SOX9 at the invasive front and an associated higher risk of relapse together with a lower risk of relapse the higher the SOX9 expression detected, could illustrate that SOX9 is crucial tumor suppressor governing proliferation and expansion of the cells. The lack of expression can be triggered by mutations but also by promoter hyper-methylations. Indeed, SOX9 promoter hyper-methylations has been previously reported to be associated with SOX9 expression loss and bladder cancer progression[26].

Decreased expression and the heterogeneous expression of the transcription factor in the colon tumors might be dependent on extracellular signaling from the stroma. It could be speculated that the tumor cells with low or absent SOX9 at the invasive are a subclone gaining properties to enter the epithelial-mesenchymal transition. Accordingly, providing the tumor cells with the ability to migrate and invade further into the tissue, thereby gaining increased metastatic potential and reaching another stage of cancer progression.

The fact that we did not find any association to risk of relapse at the luminal surface of the neoplastic glands could be that loss of SOX9 in the tumor cells of this area does not have the same metastatic potential as deeper in the tissue. However, further studies are necessary to shed light on the exact functions of the epigenetic and mutational effects on SOX9 protein expression and the potential association to cancer progression and risk of relapse. 
SOX9 has previously been reported as overexpressed in colorectal cancer, which we also observed in the majority of the cases[7,15,25,27-32].Currently, there is no consensus guidelines nationally or internationally regarding scoring of SOX9. Thus, we decided to score most informatory by scoring at the invasive front and at the luminal surface instead of an average score of the whole tumor slide. However, this also makes our study differ from other prognostic studies of SOX9 in colorectal cancer $[7,15,30]$.

We did not find any correlation between high expression of SOX9 and poorer prognosis as previously reported[15] nor could Panza et al. in their study[30]. The description of the patient cohort is sparse which makes it difficult to point out differences from the present study and thereby plausible explanations for the discrepancies of the studies. Interestingly, SOX9 protein overexpression has been found to predict reduced overall survival in stage III and not stage II colon cancer patients, which provides a plausible explanation to why we did not find any correlation between SOX9 expression and overall survival[27]. Furthermore, the use of different antibodies can influence the results, as these potentially recognize different epitopes of the SOX9 protein. This also constrains our study, as we only investigated one antibody which was the most applied in the literature. Optimally, we could have investigated and validated our findings more thorough by applying antibodies from additional suppliers but unfortunately this was not possible.

Both high and low SOX9 expression levels has been associated with MSI[30,31]. However, we did not find any correlation between SOX9 expression levels and MMR status. As expected dMMR in our study was significant associated with a lower risk of relapse which has also been reported by other studies[33,34]. The majority of the dMMR tumors were hyper-methylated in the MLHI promoter, indicating sporadic colon cancer[6]. MMR deficiency was investigated by IHC as part of the diagnostic routine. The MMR deficiency could have been verified by MSI testing, however this was unfortunately not possible. Instead the methylation profile of the MMR genes was tested to provide additional information to the cohort as $M L H 1$ promoter hyper-methylations are most 
commonly found in sporadic colon cancer cases. However, it should be noted that a few cases of patients harboring both $M L H 1$ promoter hyper-methylations and $M L H 1$ mutations has been reported[6,35]. The likeliness of this is small in our study as we only selected patients at 50 years of age or above. It is uncertain whether the patients with dMMR tumors without hyper-methylations had a hereditary factor. Unfortunately, in our study these tumors were not genetically investigated as this has not been the general procedure.

The SOX9 expression level was neither associated with gender nor age as others have previously described[30]. Of the conventional histopathological risk factors vein infiltration and the histological subtypes were not associated with relapse which may be due to the relatively small size of our study.

An important limitation of this study is the exclusion of patients with other primary cancers prior to or after their primary diagnosis of stage II colon cancer. This comprises a selection bias of the patients not reflecting all patients seen in the clinic. Due to the setup of the study we cannot argue that our findings can be directly translated into recommending adjuvant therapy to SOX9 expression indicated high risk patients. We believe that a larger randomized clinical trial is needed for answering that present issue. However, the stratification of high risk patients by their SOX9 expression may provide information for more intense follow-up of these patients and we believe that a hazard ratio of 2.32 would be high enough to justify this.

\section{Conclusion}

In conclusion this is the first study to report that low SOX9 protein expression at the invasive front of primary stage II colon tumors predicts high risk of relapse. Conversely, a high SOX9 protein expression level at the invasive front of primary stage II colon tumors predicts low risk of relapse. SOX9 expression may have an important value as a biomarker for prediction of relapse. If our 
findings are further validated, SOX9 analysis has a potential to enter the clinical pathology routine in evaluating risk of relapse for further personalized treatment.

\section{Abbreviations}

dMMR, Mismatch repair deficiency; IHC, Immunohistochemistry; MLH1, MutL homolog 1 ; MMR, Mismatch repair; MSH2, MutS protein homolog 2; MSH6, MutS homolog 6; MSI, Microsatellite instability; pMMR, Mismatch repair proficient; PMS2, Postmeiotic Segregation Increased 2; SOX9, Sex-determining region y-box 9; TMA, Tissue microarray

\section{Acknowledgements}

The work was funded by Department of Pathology at Herlev University Hospital, Department of Science, Systems and Models at Roskilde University, Familien Spogaards Fund, Thora og Viggo Groves Mindelegat, Direktør Jacob Madsen \& Hustru Olga Madsens Fund, and Dagmar Marshalls Fund. 


\section{References}

[1] Jemal A, Bray F, Center MM, Ferlay J, Ward E, Forman D. Global cancer statistics. CA Cancer J Clin 2011;61:69-90.

[2] Benson III AB, Schrag D, Somerfield MR, et al. American Society of Clinical Oncology Recommendations on Adjuvant Chemotherapy for Stage II Colon Cancer. J Clin Oncol 2004;22:3408-19.

[3] Böckelman C, Engelmann BE, Kaprio T, Hansen TF, Glimelius B. Risk of recurrence in patients with colon cancer stage II and III : A systematic review and meta-analysis of recent literature. Acta Oncol (Madr) 2015;54:5-16.

[4] Hanahan D, Weinberg RA. Hallmarks of cancer: The Next Generation. Cell 2011;144:646-74.

[5] Peña-Diaz J, Jiricny J. Mammalian mismatch repair: Error-free or error-prone? Trends Biochem Sci 2012;37:206-14.

[6] Parsons MT, Buchanan DD, Thompson B, Young JP, Spurdle AB. Correlation of tumour BRAF mutations and MLH1 methylation with germline mismatch repair (MMR) gene mutation status: a literature review assessing utility of tumour features for MMR variant classification. J Med Genet 2012;49:151-7.

[7] Matheu A, Collado M, Wise C, et al. Oncogenicity of the Developmental Transcription Factor Sox9. Cancer Res 2012;72:1301-15.

[8] Mori-Akiyama Y, van den Born M, van ES JH, et al. SOX9 is Required for the Differentiation of Paneth Cells in the Intestinal Epithelium. Gastroenterology 2007;133:539-46.

[9] Blache P, van de Wetering M, Duluc I, et al. SOX9 is an intestine crypt transcription factor, is regulated by the Wnt pathway, and represses the CDX2 and MUC2 genes. J Cell Biol 2004;166:3747.

[10] Bastide P, Darido C, Pannequin J, et al. Sox9 regulates cell proliferation and is required for Paneth cell differentiation in the intestinal epithelium. J Cell Biol 2007;178:635-48.

[11] Poulsen TS, Espersen MLM, Kofoed V, Dabetic T, Høgdall E, Balslev E. Comparison of Fluorescence In Situ Hybridization and Chromogenic In Situ Hybridization for Low and High Throughput HER2 Genetic Testing. Int J Breast Cancer 2013;2013:1-5.

[12] Lan KC, Chen YT, Chang C, et al. Up-Regulation of SOX9 in Sertoli Cells from Testiculopathic Patients Accounts for Increasing Anti-Mullerian Hormone Expression via Impaired Androgen Receptor Signaling. PLoS One 2013;8:1-11. 
[13] Sashikawa Kimura M, Mutoh H, Sugano K. SOX9 is expressed in normal stomach, intestinal metaplasia, and gastric carcinoma in humans. J Gastroenterol 2011;46:1292-9.

[14] Sun M, Uozaki H, Hino R, et al. SOX9 expression and its methylation status in gastric cancer. Virchows Arch 2012;460:271-9.

[15] Lü B, Fang Y, Xu J, et al. Analysis of SOX9 Expression in Colorectal Cancer. Am J Clin Pathol 2008;130:897-904.

[16] Ramalingam S, Daughtridge GW, Johnston MJ, Gracz AD, Magness ST. Distinct levels of Sox9 expression mark colon epithelial stem cells that form colonoids in culture. Am J Physiol Gastrointest Liver Physiol 2012;302:G10-20.

[17] Chakravarty G, Moroz K, Makridakis NM, et al. Prognostic significance of cytoplasmic SOX9 in invasive ductal carcinoma and metastatic breast cancer. Exp Biol Med 2011;236:145-55.

[18] Bosman FT, Carneiro F, Hruban RH, Theise ND. WHO Classification of Tumours of the Digestive System. 4th ed. Lyon: IARC Press; 2010.

[19] Kaae J, Szecsi PB, Meldgaard M, et al. Individuals with complete filaggrin deficiency may have an increased risk of squamous cell carcinoma. Br J Dermatol 2014;170:1380-1.

[20] McShane LM, Altman DG, Sauerbrei W, Taube SE, Gion M, Clark GM. REporting recommendations for tumor MARKer prognostic studies (REMARK). Eur J Cancer 2005;41:1090-6.

[21] Pevny LH, Lovell-Badge R. Sox genes find their feet. Curr Opin Genet Dev 1997;7:338-44.

[22] Marcker Espersen ML, Olsen J, Linnemann D, Høgdall E, Troelsen JT. Clinical Implications of Intestinal Stem Cell Markers in Colorectal Cancer. Clin Colorectal Cancer 2015;14:63-7.

[23] The Cancer Genome Atlas Network. Comprehensive Molecular Characterization of Human Colon and Rectal Cancer. Nature 2012;487:330-7.

[24] Zalzali H, Naudin C, Bastide P, et al. CEACAM1, a SOX9 direct transcriptional target identified in the colon epithelium. Oncogene 2008;27:7131-8.

[25] Abdel-Samad R, Zalzali H, Rammah C, et al. MiniSOX9, a dominant-negative variant in colon cancer cells. Oncogene 2011;30:2493-503.

[26] Aleman A, Adrien L, Lopez-Serra L, et al. Identification of DNA hypermethylation of SOX9 in association with bladder cancer progression using CpG microarrays. Br J Cancer 2008;98:466-73.

[27] Candy PA, Phillips MR, Redfern AD, et al. Notch-induced transcription factors are predictive of survival and 5-fluorouracil response in colorectal cancer patients. Br J Cancer 2013;109:1023-30. 
[28] Lü B, Xu J, Lai M, Zhang H, Chen J. A transcriptome anatomy of human colorectal cancers. BMC Cancer 2006;6:1-9.

[29] Huang M-Y, Chen H-C, Yang I-P, et al. Tumorigenesis and tumor progression related gene expression profiles in colorectal cancer. Cancer Biomarkers 2013;13:269-79.

[30] Panza A, Pazienza V, Ripoli M, et al. Interplay between SOX9, $\beta$-catenin and PPAR $\gamma$ activation in colorectal cancer. Biochim Biophys Acta 2013;1833:1853-65.

[31] Andersen CL, Christensen LL, Thorsen K, et al. Dysregulation of the transcription factors SOX4, CBFB and SMARCC1 correlates with outcome of colorectal cancer. Br J Cancer 2009;100:511-23.

[32] Shen Z, Deng H, Fang Y, et al. Identification of the interplay between SOX9 and S100P in the metastasis and invasion of colon carcinoma. Oncotarget 2015;6:20672-84.

[33] Sinicrope FA, Foster NR, Thibodeau SN, et al. DNA Mismatch Repair Status and Colon Cancer Recurrence and Survival in Clinical Trials of 5-Fluorouracil-Based Adjuvant Therapy. J Natl Cancer Inst 2011;103:863-75.

[34] Hutchins G, Southward K, Handley K, et al. Value of mismatch repair, KRAS, and BRAF mutations in predicting recurrence and benefits from chemotherapy in colorectal cancer. J Clin Oncol 2011;29:1261-70.

[35] de la Chapelle A, Hampel H. Clinical Relevance of Microsatellite Instability in Colorectal Cancer. J Clin Oncol 2010;28:3380-7. 


\section{Figure legends}

Figure 1. Flow-chart showing enrollment and exclusion of patients from the study. Abbreviations: n, number of patients; IBD, inflammatory bowel diseases.

Figure 2. Immunohistochemical staining of SOX9 in normal colon tissue (A,B) and in stage II colon cancer tissue (C, D, E, F). (A) SOX9 expression by an x2.7 magnification. (C, E) SOX9 expression by an x1.25 magnification. (B, D, F) are x20 magnification of marked areas of (A, C, E). $(A, B)$ SOX9 is expressed in nuclei of the epithelial cells at the bottom of the crypts. (C, D) High expression of SOX9 in tumor cells. (E, F) Undetected SOX9 expression in tumor cells, including internal stromal control for the SOX9 staining.

Figure 3. Relapse free survival of low vs. high SOX9 expression at (A) the luminal surface and at

(B) the invasive front of the primary tumors from patients with stage II colon cancer. Abbreviations:

HR, Hazard ratio (Cox proportional hazards model, univariate); CI, Confidence interval. 
Consecutively included stage II colon cancer

patients registered in the national pathology registry

of Denmark from January 2005 to August 2008

$(\mathrm{N}=282)$

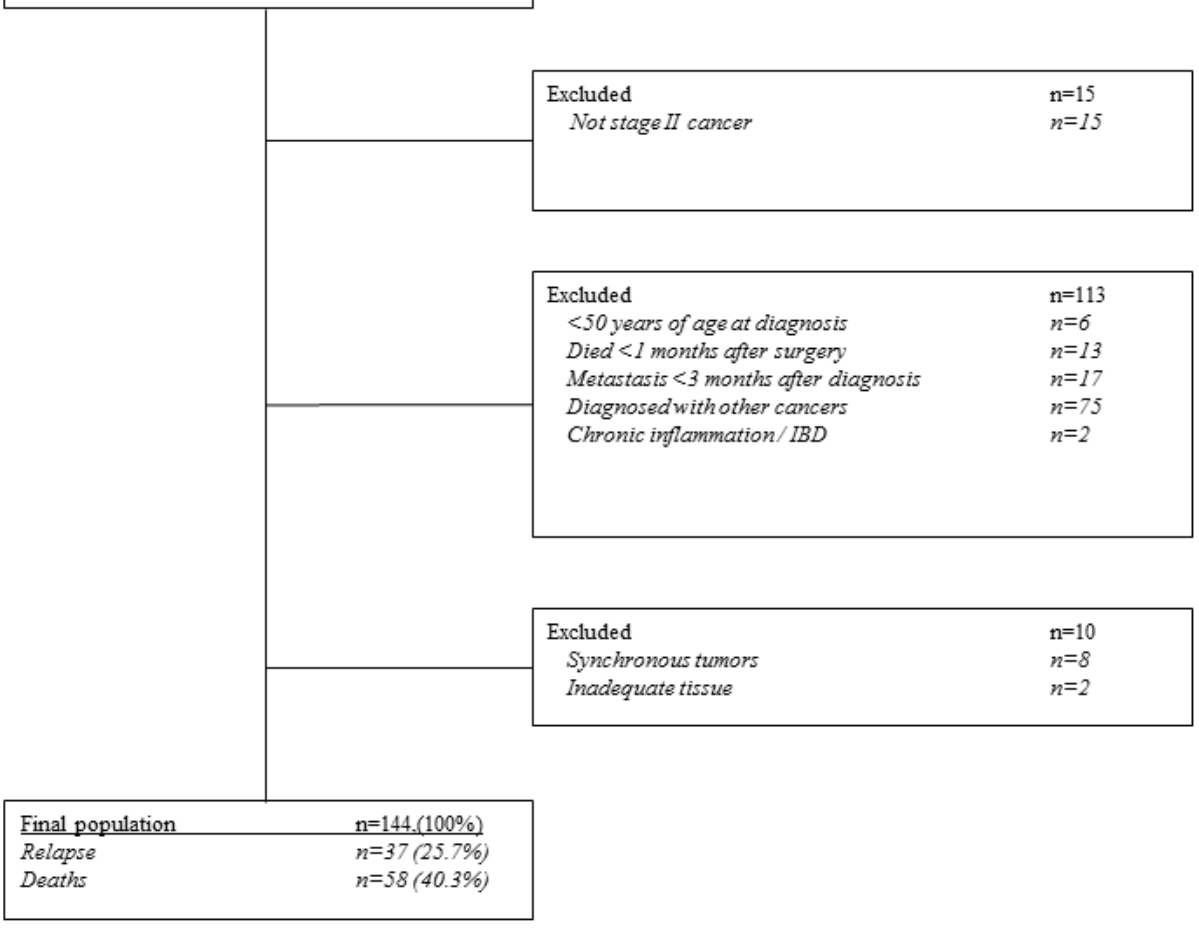

Figure 1 
A

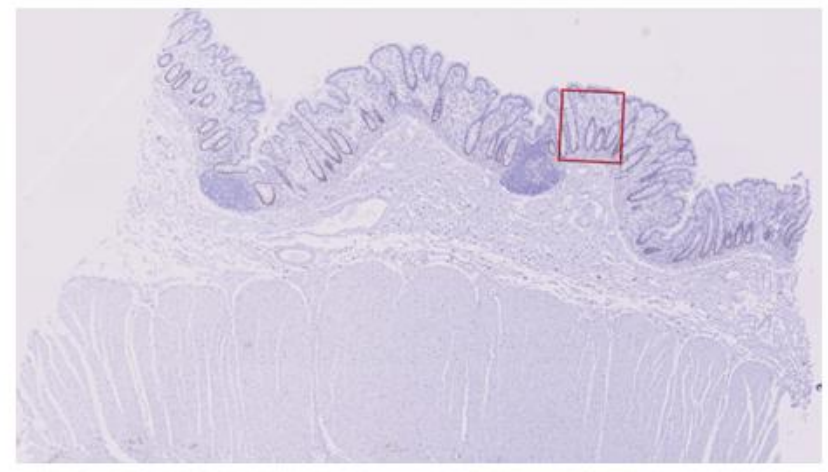

C
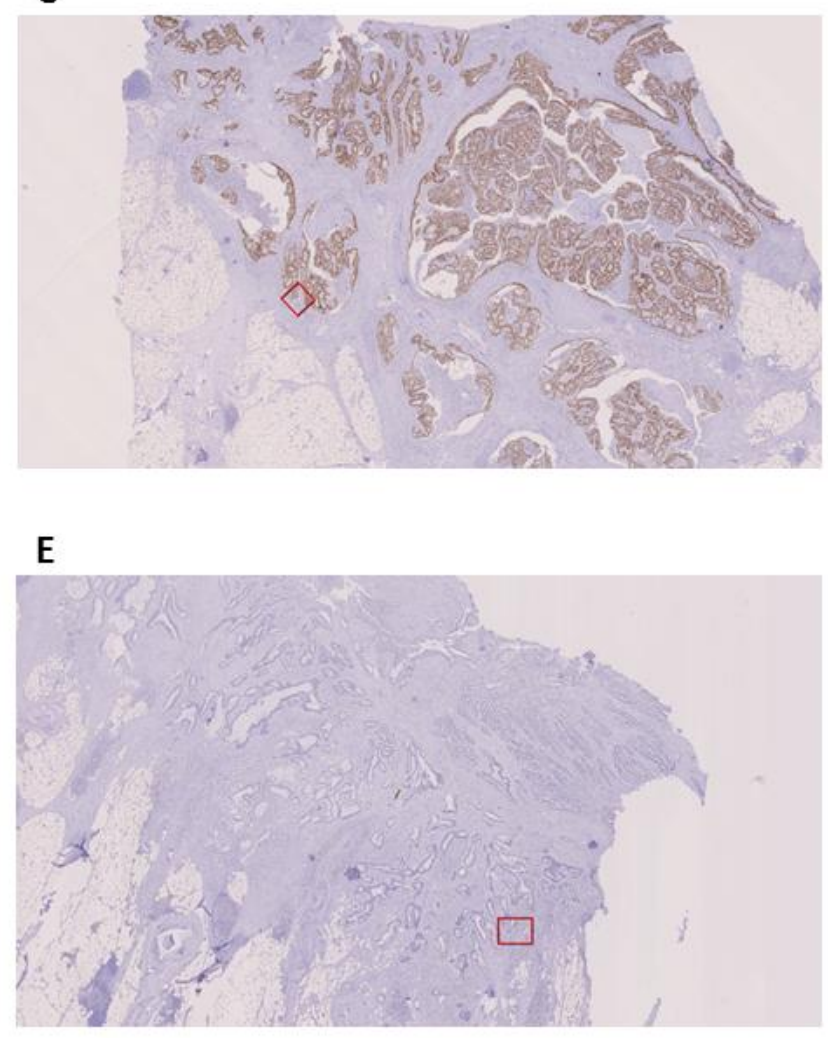

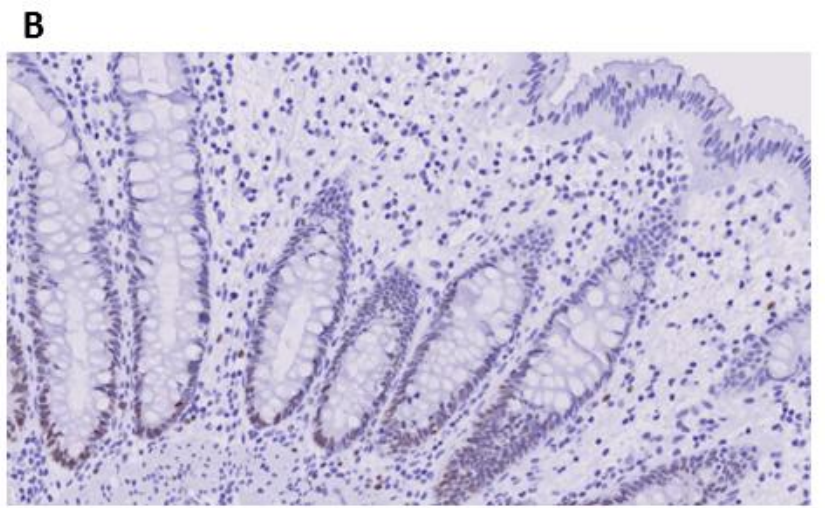

\section{D}

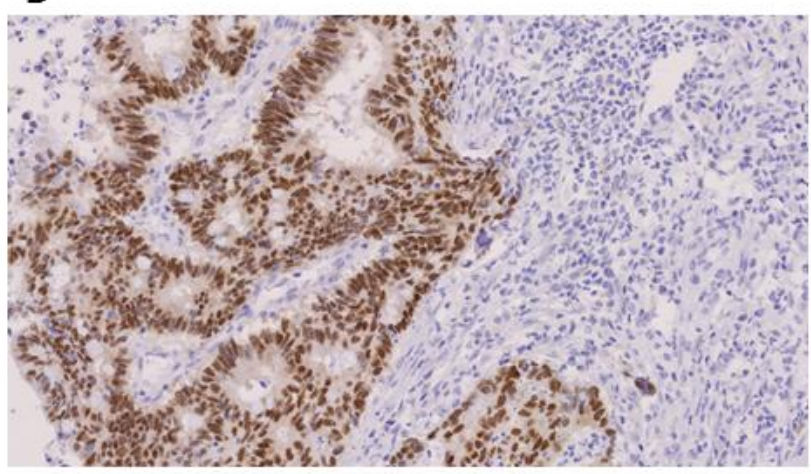

F

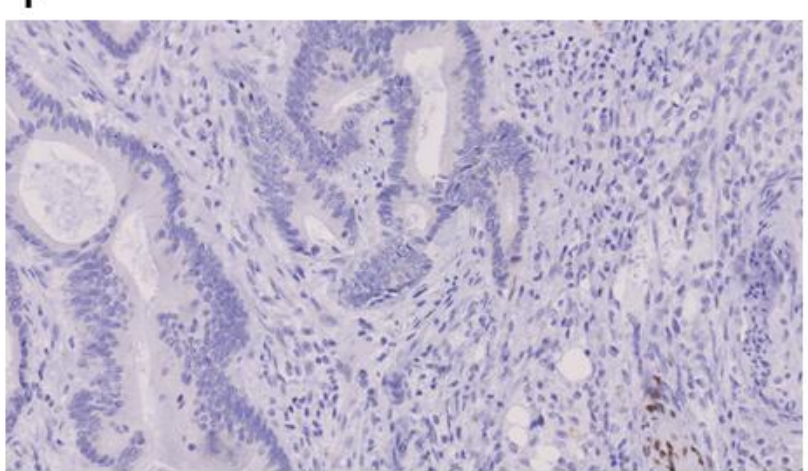

Figure 2 
A

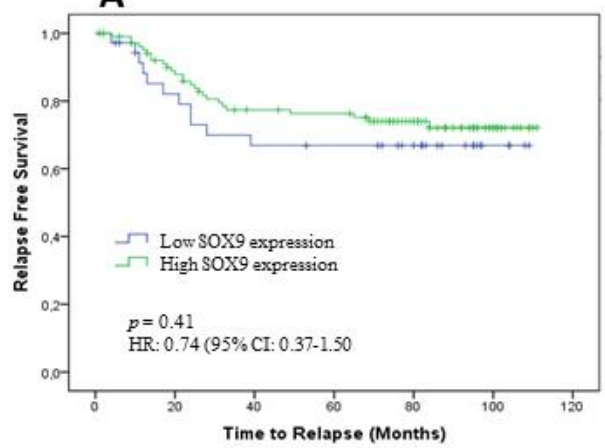

B

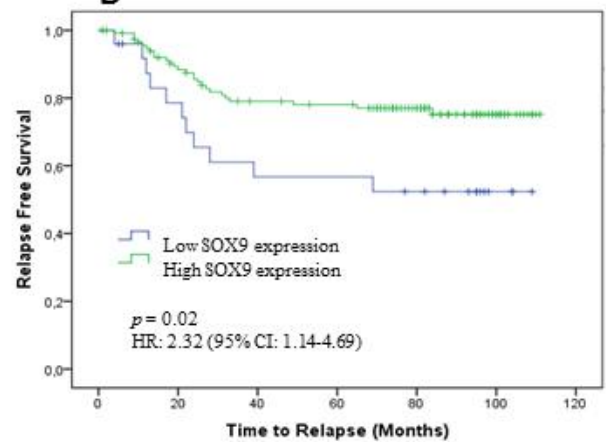

Figure 3 


\section{Tables}

Table 1. Patient baseline characteristics.

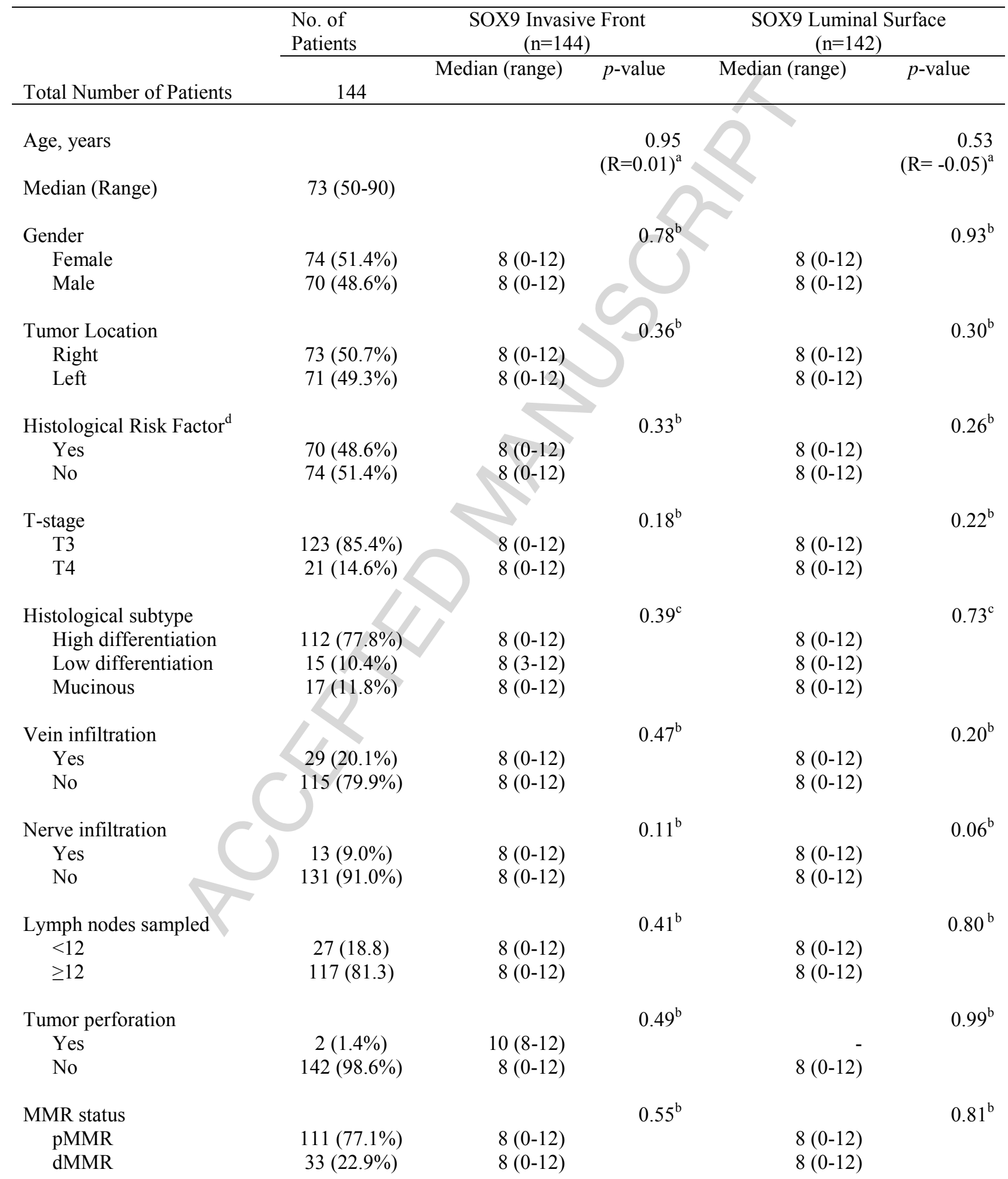

${ }^{\mathrm{a}}$ Spearman Rank correlation; ${ }^{\mathrm{b}}$ Mann-Whitney U test; ${ }^{\mathrm{c}}$ Kruskal-Wallis test; ${ }^{\mathrm{d}}$ The patient was considered to have a histopathological risk factor if either of the risk factors was present in the tumor: T4 stage, nerve and/or vein infiltration, low differentiated histology (unless the tumor was dMMR), tumor perforation, or less than 12 lymph nodes sampled at primary resection; Left sided tumors includes tumors of left flexur, descendens, sigmoideum. Right sided tumor includes tumor of cecum, ascendens, right flexur, and transversum. Abbreviations: dMMR, Mismatch repair deficient; MMR, Mismatch Repair; n, number of patients analyzed; pMMR, 
Mismatch repair proficient; SOX9 Invasive front, SOX9 expression at the invasive front of the tumor; SOX9 luminal surface, SOX9 expression at the luminal surface of the neoplastic glands.

Table 2. Univariate and multivariate analysis of prognostic factors for relapse.

\begin{tabular}{lccc} 
& \multicolumn{2}{c}{ Univariate Analysis } & Multivariate Analysis \\
\hline Variable & Hazard Ratio & $p$-value & Hazard Ratio \\
& $(95 \% \mathrm{CI})$ & & $(95 \% \mathrm{CI})$
\end{tabular}

\section{Gender}

Male

Female

Tumor Location

$\begin{array}{lll}\text { Left } & 1.47(0.76-2.83) & 0.25 \\ \text { Right } & \end{array}$

SOX9 Expression at the Invasive Front

Continuous score
High
Low

$$
\begin{array}{ll}
0.73(0.56-0.94) & 0.01^{\mathrm{a}} \\
2.32(1.14-4.69) & 0.02
\end{array}
$$$$
0.75(0.58-0.96)^{\mathrm{a}}
$$$$
0.02^{\mathrm{a}}
$$$$
2.32(1.14-4.69)^{b}
$$

MMR Status
dMMR
$0.19(0.05-0.80)$
0.02
$0.24(0.06-0.99)^{\mathrm{a}}$
$0.05^{\mathrm{a}}$
pMMR

Histopathological Risk Factor ${ }^{\mathrm{c}}$

$$
\begin{aligned}
& \text { Yes } \\
& \text { No }
\end{aligned}
$$$$
3.01(1.48-6.08)
$$$$
<0.01
$$$$
2.65(1.31-5.39)^{a}
$$$$
2.7(1.32-5.44)^{b}
$$$$
0.01^{\mathrm{a}}
$$

Histological subtype

High differentiation Low differentiation Mucinous
$0.705(0.29-1.70) \quad 0.44$

$0.46(0.93-2.28) \quad 0.46$

$1.21(0.55-2.64) \quad 0.64$

Yes

No

Yes

No

4.45 (1.94-10.20)

$<0.01$

Lymph nodes sampled

${ }^{\mathrm{a}}$ Cox proportional hazards model with SOX9 expression as a continuous score. The hazard ratio is based of a difference of 3 in the SOX9 score; ${ }^{b}$ Cox proportional hazards model with SOX9 expression as a dichotomous score (High/Low). ${ }^{\mathrm{c}}$ If either of the risk factors was present in the tumor: T4 stage, nerve and/or vein infiltration, low differentiated histology (unless the tumor was dMMR), tumor perforation, or less than 12 lymph nodes sampled at primary resection, the patient was considered to have a histopathological risk factor. Abbreviations: CI, Confidence interval; dMMR, Mismatch repair deficiency; MMR, Mismatch repair; pMMR, Mismatch repair proficient. 